\title{
Hybridization of GNSS and On-Board Sensors for Validating the Aurora Ecosystem
}

\author{
Martti Kirkko-Jaakkola, Simo Marila, Sarang Thombre, Salomon Honkala, Hannu Koivula, Heidi Kuusniemi, \\ Finnish Geospatial Research Institute, National Land Survey of Finland
}

Stefan Söderholm, HERE Technologies

\section{BIOGRAPHIES}

Martti Kirkko-Jaakkola received his D.Sc. (Tech.) degree from Tampere University of Technology, Finland, in 2013. Currently, he is a Research Manager and the leader of the Sensors and Indoor Navigation research group at the Finnish Geospatial Research Institute (FGI). His research interests include precise satellite positioning and timing, low-cost motion sensors, and indoor navigation.

Simo Marila received his Master of Science degree in 2011 from Aalto University, Finland. His main subject was geodesy. He works now as a Research Scientist at the Department of Geodesy and Geodynamics in FGI. He was involved in renewing the Finnish permanent GNSS network, and his recent research work has been mainly related to the reliability and accuracy of GNSS positioning.

Sarang Thombre received his Doctoral degree from Tampere University of Technology in 2014. He works as a Research Manager and Research Group Leader at FGI. His research interests include positioning receiver implementation and performance validation, interference studies, and localization for autonomous vehicles.

Salomon Honkala received the M.Sc. (Tech.) degree in electrical engineering from Aalto University, Espoo, Finland, in 2016. He is a Ph.D. candidate at Tampere University, Finland. His current research interests include GNSS vulnerabilities, software GNSS receiver design, interference mitigation, GNSS timing robustness, precise positioning, and Arctic navigation.

Hannu Koivula is the leader of the Reference Systems research group at the FGI. He received his M.Sc. (tech) degree in 1994 (Helsinki University of Technology) and Lic. (tech) degree in 2007 (Aalto University), both majoring in geodesy. He has 20 years of research experience in high accuracy GNSS applications and GNSS networks. He has studied the accuracy of GNSS applications and lately specialized in metrological traceability of GNSS measurements.

Stefan Söderholm received the M.Sc. degree from the Department of Experimental Physics, Åbo Akademi University, Turku, Finland, in 1991, and the Licentiate degree from the Department of Applied Physics, University of Turku, Finland, in 1996. He is currently the GNSS Team Leader with HERE Technologies, Tampere, Finland, where he is heading the companies work on improving GNSS accuracy and reliability, which includes algorithm development and product specifications within the technology areas of precise point positioning, anti-jamming, and anti-spoofing. His current research interests include multi-GNSS receiver design, BOC signal processing, GNSS spoofing and interference detection, and PPP/RTK methods.

Heidi Kuusniemi has worked in research and development within positioning technologies for over 18 years. She is currently a Research Professor of satellite navigation with FGI. She is also the Director of the multi-disciplinary research platform digital economy at the University of Vaasa, Finland. She is also an Adjunct Professor in satellite navigation and positioning technologies at Tampere University, Finland, and Aalto University, Espoo, Finland. She is also the President of the Nordic Institute of Navigation. Her current research interests include GNSS reliability monitoring, GNSS interference detection and mitigation, self-contained sensors, indoor positioning, Internet of Things, and the digital economy. 


\begin{abstract}
This paper presents a hybrid navigation algorithm based on loose coupling of the on-board speedometer and inertial sensors of a land vehicle with a GNSS receiver. An Extended Kalman Filter estimating ten error states is used as the hybridization framework. The algorithm is developed to serve as a baseline for the evaluation of the navigation infrastructure of the Aurora ecosystem which is an Arctic test bed for autonomous vehicles and intelligent transport systems. In the experimental tests we focus on the performance of the navigation algorithm during GNSS outages. First, the tests indicate that the quality of GNSS updates has an immediate effect on how fast the position errors accumulate when GNSS becomes unavailable. Second, using low-cost sensors together with the current navigation infrastructure available at the Aurora test site, GNSS position fixes need to be obtained at intervals no longer than 4 seconds in order to maintain a $95 \%$ horizontal positioning accuracy better than 0.2 meters. The results serve as a basis for recommendations for further development of the Aurora ecosystem, suggesting that further positioning infrastructure could be deployed for guaranteeing a navigation performance adequate for autonomous vehicles.
\end{abstract}

\title{
INTRODUCTION
}

Traditionally, sub-meter positioning accuracies have mostly been required by professional users such as surveyors and precision farmers. However, with the growing interest in intelligent transport system (ITS) technology and autonomous vehicles, such needs are going to become more common. It is evident that autonomous driving requires a high positioning performance, but no standard specifications have been established. A set of requirements is proposed in [1] as a synthesis of interviews and existing literature. In [2], a horizontal positioning accuracy requirement of $0.5 \mathrm{~m}(95 \%)$ is postulated; an accuracy specification in the order of $0.1-$ $0.3 \mathrm{~m}$ is foreseen in [3].

It is well known that global navigation satellite systems (GNSS) cannot meet these accuracy requirements alone, but together with correction data they can be achieved by means of real-time kinematic (RTK) or precise point positioning (PPP) methods. Unfortunately, accuracy is not the only key performance indicator: in particular, GNSS cannot guarantee a $100 \%$ position solution availability because of its sensitivity to environmental factors such as signal blockage and radio frequency interference. Furthermore, GNSS receivers typically provide an update rate in the order of 1 or $10 \mathrm{~Hz}$, which can be insufficient for tracking vehicle dynamics at high velocities.

The problem of position availability and update rate can be solved using on-board motion sensors together with GNSS. Given the nonholonomic motion constraint, the trajectory of a land vehicle is straightforward to estimate based on measurements from a speedometer, inertial sensors, magnetometer, etc. [4]. This approach has been shown feasible for the mitigation of GNSS interference and spoofing [5, 6], and commercial hybrid navigation solutions targeting autonomous vehicles have been released on the market [7]. In addition, imaging sensors such as cameras and laser scanners can provide motion information. The incorporation of motion sensors to navigation introduces two challenges: First, the sensors need to be calibrated for biases, scale factors, etc. Second, they do not produce absolute position information but only relative increments to previous locations, implying that the accuracy degrades over time in the absence of GNSS updates. Consequently, given the vulnerabilities of GNSS, additional infrastructure may be needed to meet the performance requirements.

With the increased interest in autonomous driving, various test beds have been built around the world to be used by developers of autonomous vehicles, ITS, and related technology, e.g., [8-10]. Typically, such test beds offer infrastructure for, at least, telecommunications and precise navigation as well as other information such as accurate maps. These test beds are ideal ecosystems for the development and evaluation of additional navigation infrastructure that can address the availability concerns of GNSS. Obviously, there is a need to validate that the test bed infrastructure can meet the performance needs of autonomous vehicles.

In this paper, we present a fusion algorithm for GNSS, inertial sensors, and a vehicle speedometer, and analyze the resulting performance in light of the proposed accuracy requirements for autonomous vehicles The algorithm is based on nonholonomic constraints, using the gyroscopes to estimate the three-dimensional attitude of the vehicle and propagating the position based on the measured speed. GNSS updates are incorporated in a loosely coupled architecture, and the initial attitude is determined based on accelerometers and GNSS-derived heading. The goal is to assess the quality of the sensor-based position estimate under degraded GNSS availability: we investigate both temporary GNSS outages and lower GNSS update frequencies. The navigation algorithm will serve as a baseline to provide recommendations for future development of the Aurora ecosystem [8] located in Northern Finland, complementing previous performance studies [11]. 
This paper is organized as follows. First, we outline the objectives of the Aurora Ecosystem in the field of autonomous driving research. Then, the hybrid navigation algorithm used for the performance validation is described. Finally, experimental results are presented to quantify the obtained performance during degraded GNSS availability.

\section{AURORA ECOSYSTEM}

The Aurora Borealis Corridor [8] is a cross-border test bed for autonomous driving, located at the E8 road in Finland and Norway (Figure 1). There are two test sections with specialized instrumentation: one on the Finnish side, called Aurora, and another on the Norwegian side of the border, called Borealis. While different test beds for autonomous driving are being deployed around the world, e.g., $[9,10]$, the purpose of the Aurora Borealis Corridor is to enable testing of autonomous vehicles in challenging Arctic winter conditions where roads are slippery, lane markings are covered by snow, the ionosphere is highly active, and it is dark for most of the day. From a positioning, navigation, and timing (PNT) point of view, it is noteworthy that the Aurora Borealis Corridor is located within the EGNOS coverage area; however, because of the high-latitude location (above $67^{\circ} \mathrm{N}$ ), the visibility of geostationary satellites in the test bed area is limited. Where necessary, the EGNOS augmentation data can be downloaded online from the EGNOS Data Access Service [12] instead of the signal-in-space.

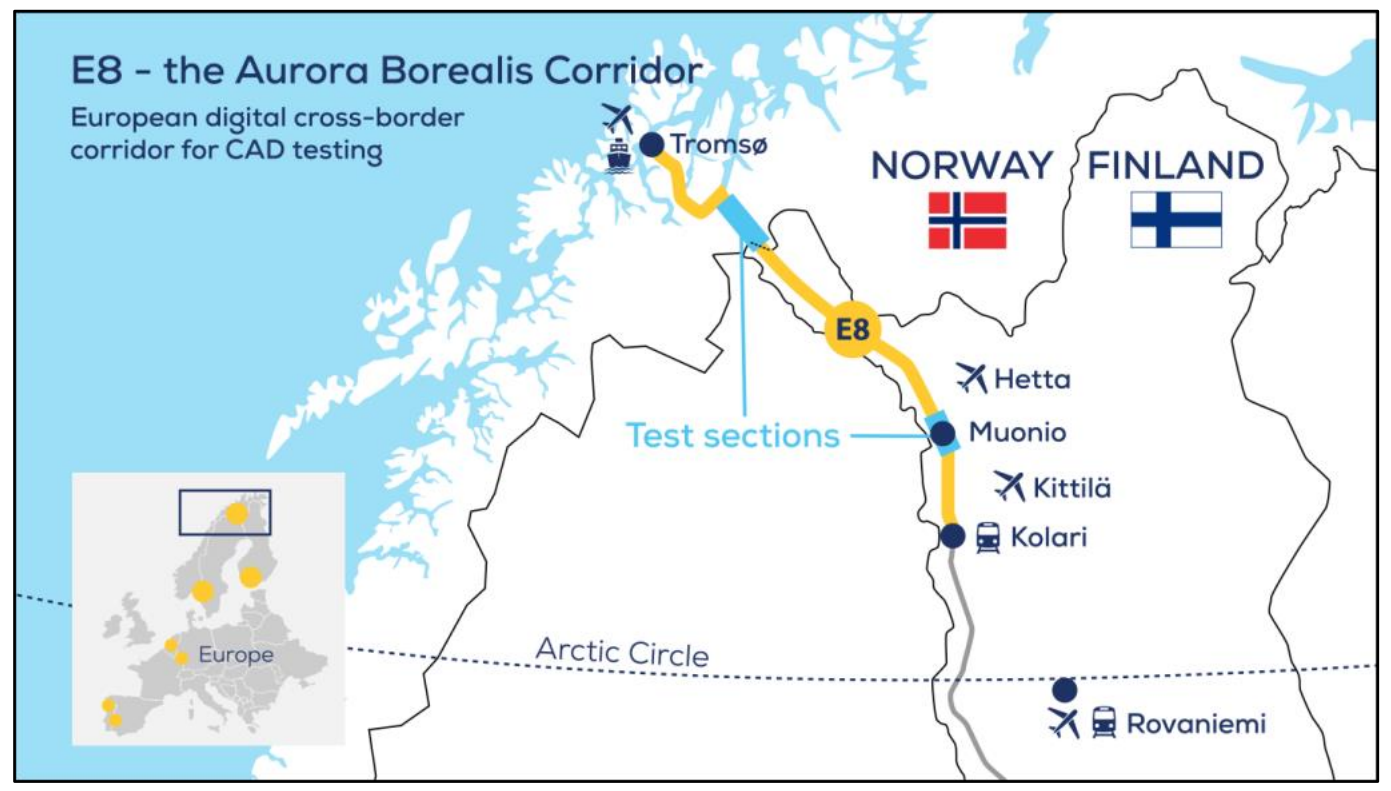

Figure 1 Map of the Aurora Borealis corridor. Image ( $)$ Finnish Transport Infrastructure Agency.

The experiments reported in this paper were conducted in the Aurora test section in Finland. For accurate PNT, the Aurora ecosystem provides various infrastructures. First, four new permanent GNSS reference stations, part of the Finnish national GNSS network FinnRef [13], have been deployed in addition to three existing FinnRef stations close to the E8 road. Using these reference stations, the FinnRef network is capable of providing precise GNSS corrections in the form of raw base station measurements or virtual reference station observations for RTK as well as state-space representation corrections for PPP. In addition, high-definition maps of the road boundaries, markings, etc. are available. The Aurora ecosystem also makes it possible for researchers and companies to deploy their proprietary technology, such as ultra-wide band radio beacons or radar reflectors, for testing in a real environment. The Aurora test bed has a good coverage of commercial cellular networks, including a dedicated 4G+ broadband network. Moreover, the Aurora ecosystem includes non-PNT related infrastructure such as roadside cameras and traffic sensors embedded in the road.

\section{HYBRIDIZATION ALGORITHM}

In this paper, we investigate sensor fusion in a loosely coupled architecture, i.e., the position solution obtained from GNSS is combined with the position estimated based on the motion sensors. The architecture is illustrated in Figure 2, with measurement inputs indicated by red color. This choice of architecture favors a low complexity of implementation over more efficient GNSS updates: whenever not enough GNSS measurements are available for position computation, an update cannot be conducted; in contrast, a 
tightly coupled system could still make use of raw measurements from less than four satellites. However, since the goal of this paper is to investigate the performance in the absence of GNSS updates, this is not a critical drawback. Furthermore, the simplicity of the architecture makes it straightforward to incorporate other sources of PNT information that can be made available at autonomous vehicle test beds, such as short-range radio ranging.

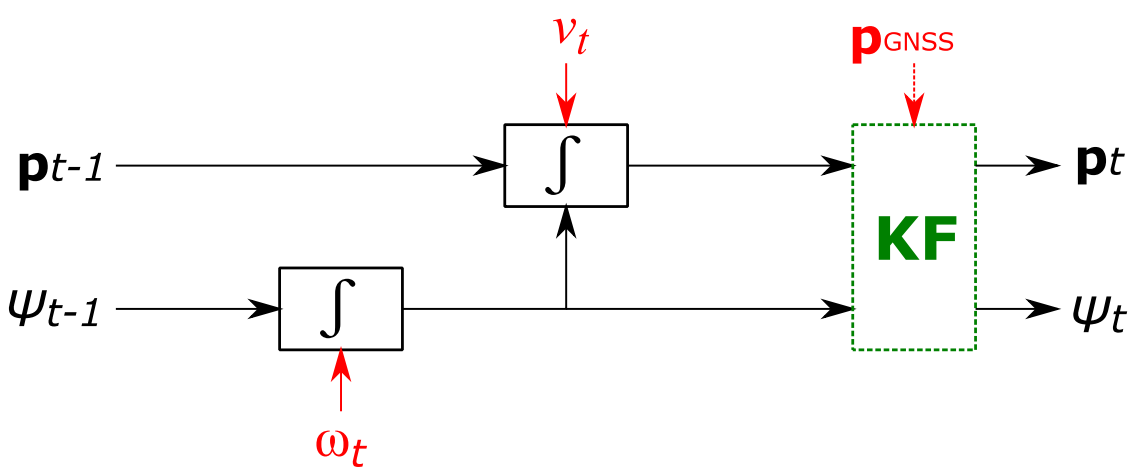

Figure 2 Architecture of the sensor fusion algorithm

In the following sections, we describe the sensor-based position equations and the Kalman Filter (KF) used for the data fusion. It is noteworthy that a two-dimensional navigation solution could be computed by only keeping track of the heading instead of the full three-dimensional attitude. However, the test site is not located on flat ground but has altitude differences up to $30 \mathrm{~m}$, rendering the validity of a two-dimensional algorithm questionable. In conjunction with map matching, a two-dimensional solution could be suitable and more robust than a solution with six degrees of freedom. However, maps are beyond the scope of this paper; they are to be investigated as future work.

\section{Position Computation}

Since motion sensors only provide relative position and attitude information, initial position and attitude estimates $\boldsymbol{p}_{t-1}$ and $\Psi_{t-1}$, respectively, are assumed available; they can be determined using GNSS and accelerometer measurements. First, the attitude $\Psi_{t-1}$ is updated using the gyroscope measurements $\omega_{t}$ following the relation

$$
\dot{\Psi}=\Psi(\omega \times)
$$

where $(\omega \times)$ denotes the $3 \times 3$ skew-symmetric cross product matrix constructed from the vector $\omega$. We use direction cosine matrices to represent the three-dimensional attitude as the rotation from the vehicle's coordinate frame to the navigation frame (Earth-centered Earth-fixed coordinate system).

Next, the position $\boldsymbol{p}_{t-1}$ is updated. Assuming the $x$-axis to be pointing forward in the vehicle's coordinate frame, the position is propagated as

$$
\boldsymbol{p}_{t}=\boldsymbol{p}_{t-1}+\frac{1}{2}\left(\Psi_{t-1}+\Psi_{t}\right)\left[\begin{array}{c}
v_{t} \\
0 \\
0
\end{array}\right] \Delta T
$$

where $v_{t}$ denotes the speed measurement and $\Delta T$ is the time step. Note that in this study, we use speed measurements from a wheel encoder instead of accelerometers in order to avoid error accumulation due to the integration of gravity compensation residuals. 
The speedometer determines the ground speed by measuring wheel rotations and applying an estimate of the tire circumference. This speed measurement is affected by two auxiliary factors. First, the instantaneous tire circumference depends on several factors such as the tread wear, tire pressure, and vehicle load, resulting in a scaling uncertainty of several percent. This scale factor $s$ is estimated on the fly by the KF and applied on the raw speedometer measurement $v_{\text {raw }}$ as follows to obtain the scale factor compensated speed $v_{S F}$ :

$$
v_{S F}=\frac{v_{r a w}}{1+s} .
$$

Second, contrary to the angular rate, the measured speed depends on the point of reference on the vehicle body. In general, the GNSS antenna and the speedometer point of reference do not coincide, resulting in a lever arm effect on the speed measurement whenever the vehicle is turning. The lever arm effect is a function of the three-dimensional vector $\boldsymbol{L}$, pointing from the speedometer reference point to the GNSS antenna, and the angular rate $\omega$, compensated for as

$$
v_{G N S S}=\left\|\left[\begin{array}{c}
v_{S F} \\
0 \\
0
\end{array}\right]-\omega \times L\right\|
$$

to obtain the GNSS antenna speed $v_{G N S S}$. The lever arm vector $\boldsymbol{L}$ has to be measured when the sensors are installed on the vehicle. As a result of the lever arm compensation, the resulting position estimates are referred to the location of the GNSS antenna.

\section{Error-State Kalman Filter}

The position and attitude propagation algorithm described above is subject to error accumulation over time. In order to mitigate the errors, an error-state KF is run. GNSS position fixes are used to estimate the contributions of the underlying uncertainty factors, after which they can be compensated for. However, GNSS updates are not available at every navigation epoch, which is why the KF block in Figure 2 is drawn with a dashed boundary.

The state vector $\boldsymbol{x}$ consists of ten components: position errors $\delta \boldsymbol{p}$, attitude errors $\delta \Psi$, gyroscope bias errors $\delta \boldsymbol{b}$, and the speedometer scale factor error $\delta s$. The state transition model can be expressed in continuous time as

$$
\dot{\boldsymbol{x}}=\left[\begin{array}{cccc}
\mathbf{0} & (\Psi \boldsymbol{v}) \times & & \Psi \boldsymbol{v}_{\text {raw }} /(1+s)^{2} \\
& \mathbf{0} & -\Psi & \\
& & \mathbf{0} & 0
\end{array}\right]\left[\begin{array}{c}
\delta \boldsymbol{p} \\
\delta \Psi \\
\delta \boldsymbol{b} \\
\delta s
\end{array}\right]=\boldsymbol{F} \boldsymbol{x}
$$

where $v=\left[\begin{array}{lll}v & 0 & 0\end{array}\right]^{T}$ and empty matrix cells correspond to zeros. It can be seen that the position errors are driven by the attitude and scale factor errors, and the attitude errors are propagated by gyro biases. The discrete-time solution of the above continuous-time system of differential equations is

$$
\boldsymbol{x}_{t}=\exp (\boldsymbol{F} \Delta T) \boldsymbol{x}_{t-1} \approx(\boldsymbol{I}+\boldsymbol{F} \Delta T) \boldsymbol{x}_{t-1}
$$

where $\boldsymbol{I}$ denotes the identity matrix. The discrete-time state transition noise covariance matrix $\boldsymbol{Q}$ is expressed as a function of the gyroscope measurement noise variance $\sigma_{g}^{2}$, speedometer measurement noise variance $\sigma_{o}^{2}$, gyroscope bias random walk noise variance $\sigma_{b}^{2}$, and speedometer scale factor random walk noise variance $\sigma_{s}^{2}$ as follows: 


$$
\boldsymbol{Q}=\left[\begin{array}{cccc}
\boldsymbol{Q}_{11} & \frac{\sigma_{g}^{2}}{2}(\Psi \boldsymbol{v}) \times \Delta T^{2} & \frac{\sigma_{s}^{2}}{2(1+s)} \Psi \boldsymbol{v} \Delta T^{2} \\
\frac{\sigma_{g}^{2}}{2}(\boldsymbol{v} \times)^{T} \Psi^{\mathrm{T}} \Delta T^{2} & \boldsymbol{Q}_{22} & -\frac{\sigma_{b}^{2}}{2} \Psi \Delta T^{2} & \\
& -\frac{\sigma_{b}^{2}}{2} \Psi^{T} \Delta T^{2} & \sigma_{b}^{2} \boldsymbol{I} \Delta T & \\
\frac{\sigma_{s}^{2}}{2(1+s)} \boldsymbol{v}^{T} \Psi^{T} \Delta T^{2} & & & \sigma_{s}^{2} \Delta T
\end{array}\right]
$$

where

$$
\begin{aligned}
& \boldsymbol{Q}_{11}=\sigma_{o}^{2} \Psi\left[\begin{array}{lll}
1 & 0 & 0 \\
0 & 0 & 0 \\
0 & 0 & 0
\end{array}\right] \Psi^{T} \Delta T+\frac{\sigma_{g}^{2}}{3}(\Psi \boldsymbol{v}) \times((\Psi \boldsymbol{v}) \times)^{T} \Delta T^{3}+\frac{\sigma_{s}^{2}}{3(1+s)^{2}} \Psi \boldsymbol{v} \boldsymbol{v}^{T} \Psi^{T} \Delta T^{3} . \\
& \boldsymbol{Q}_{22}=\sigma_{g}^{2} \boldsymbol{I} \Delta T+\frac{\sigma_{b}^{2}}{3} \Psi \Psi^{T} \Delta T^{2}
\end{aligned}
$$

This model allows for evaluating the KF state transition equations. Whenever a GNSS position fix is available, a filter update is carried out. The update takes the difference of the position estimated by the navigation algorithm and the GNSS-based position as the measurement, with the Jacobian matrix $\boldsymbol{H}$ expressed simply as

$$
\boldsymbol{H}=\left[\begin{array}{llll}
\boldsymbol{I} & \mathbf{0} & \mathbf{0} & \mathbf{0}
\end{array}\right],
$$

and the measurement noise covariance matrix defined based on precision of the GNSS fix. It is straightforward to include GNSS velocity estimates into the updates if desired; however, they were not applied in this paper.

After the KF update, the estimated error states are fed back to the navigation variables as

$$
\begin{aligned}
& \boldsymbol{p}_{t}=\boldsymbol{p}_{t}-\delta \boldsymbol{p} \\
& \Psi_{t}=(\boldsymbol{I}+\delta \Psi \times) \Psi_{t} \\
& \boldsymbol{b}_{t}=\boldsymbol{b}_{t}+\delta \boldsymbol{b} \\
& s_{t}=s_{t}+\delta s
\end{aligned}
$$

and finally, the KF state vector is reset to zero (while the covariance matrix remains intact).

\section{EXPERIMENTS}

We analyze the behavior of the implemented hybrid navigation algorithm using three different GNSS data sets: one using EGNOS corrections, another one which is an un-assisted multi-GNSS (GPS-Galileo-GLONASS-BeiDou) solution, and an RTK solution. The trajectory in each data set is a round-trip drive in the Aurora test area starting from the South. The purpose of the analysis is to characterize the position error accumulation between GNSS position updates. First, we investigate how the behavior of the errors under prolonged GNSS outages, under two different receiver processing configurations: un-assisted multi-GNSS and EGNOS. Then, we use the RTK data to find out the maximum GNSS update period where the horizontal positioning accuracy can be maintained below $20 \mathrm{~cm}$. 


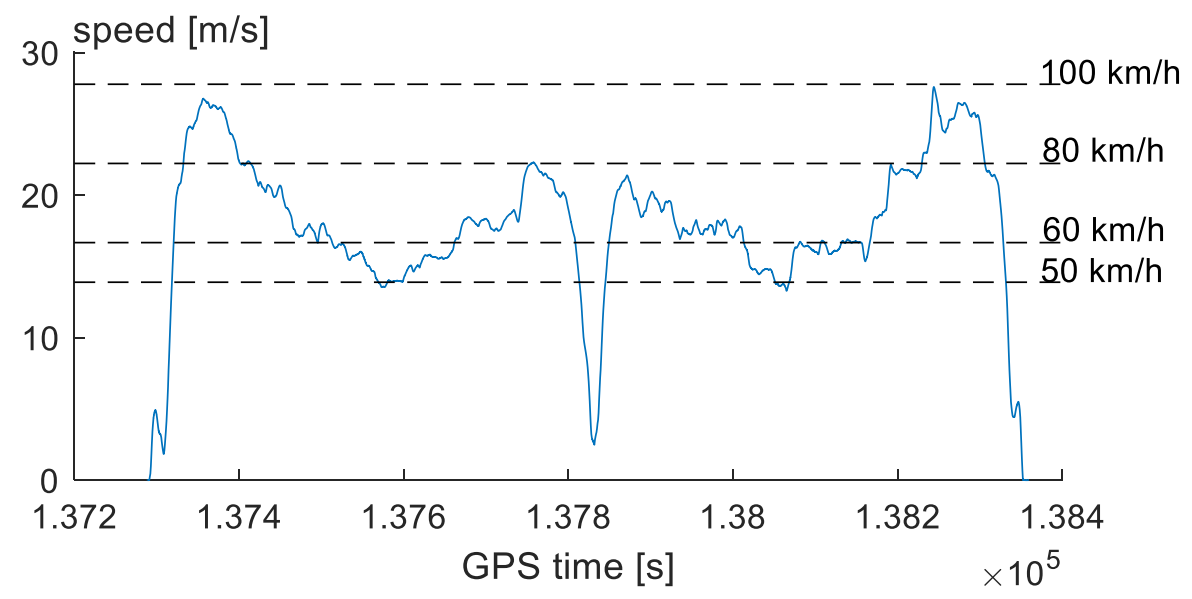

Figure 3 Example vehicle speed profile during a round-trip drive in Aurora. The horizontal dashed lines correspond to different speed limits.

It is noteworthy that during the data collection, the vehicle was driven at the same speed as other traffic on the public road. The speed limits within the Aurora test environment range from $50 \mathrm{~km} / \mathrm{h}$ (Muonio population centre) to $100 \mathrm{~km} / \mathrm{h}$, and an example speed profile is plotted in Figure 3. The higher the speed, the faster the position error accumulation; this, as well as possible differences in the choice of sensors, should be kept in mind when comparing the results with other studies.

\section{Test Setup}

The experiments were conducted using the experimental autonomous vehicle developed by the RobotCar Crew of VTT Technical Centre of Finland (Figure 4). Although the vehicle is capable of driving autonomously, this feature was not enabled in these experiments but the car was steered manually by a human driver.
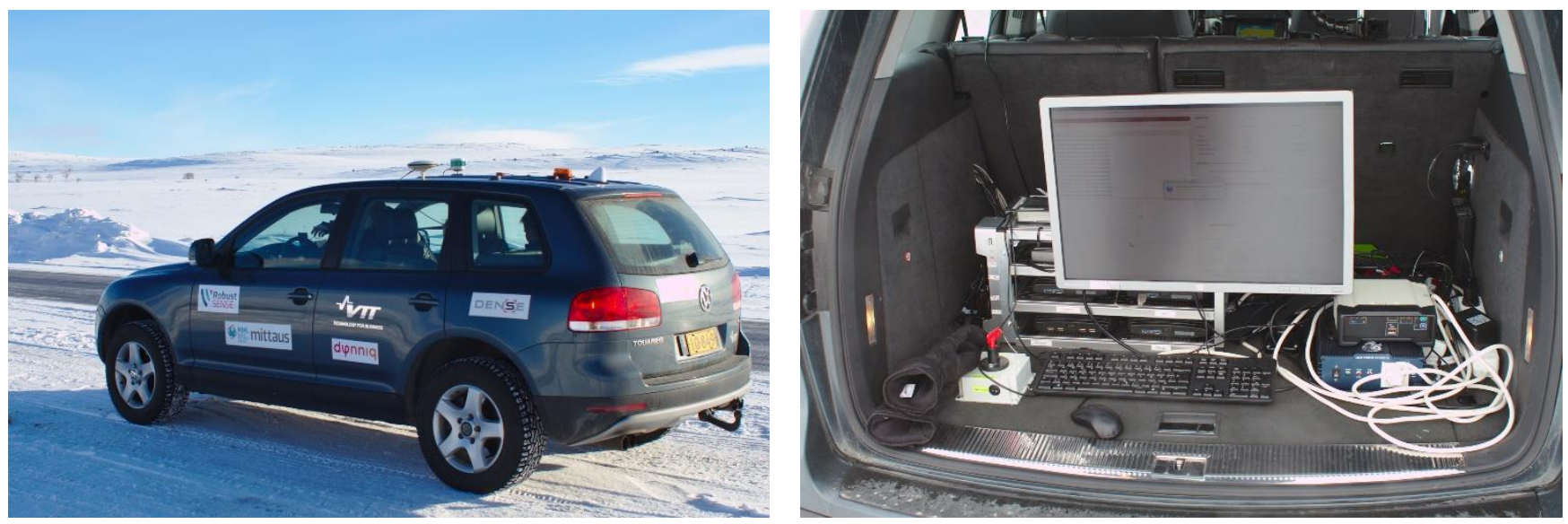

Figure 4 Test vehicle. Left: side view; right: instruments mounted in the cargo area.

A GNSS antenna was mounted on the roof of the test vehicle, and the incoming signal was split for several receivers. One of the receivers was a NovAtel Propak6 coupled with a Honeywell HG1700-AG58 IMU whose purpose was to provide an uninterrupted position and attitude reference at $100 \mathrm{~Hz}$ update rate. The other receivers were Javad Delta units configured to output position solutions at $1 \mathrm{~Hz}$ update rate using different GNSS correction data: one running in stand-alone mode, another one using EGNOS, and a third receiver using RTK corrections. Furthermore, an Xsens MTi-G-700 MEMS IMU was used to obtain the inertial data for the hybridization algorithm, and the measurements of the on-board speedometer were logged. With all the necessary data collected, the hybridization filter was run post-mission. 
The lever arm vector between the IMU and the GNSS antenna reference point was surveyed beforehand using a total station. In addition, the coordinates of each wheel were measured in the same coordinate frame such that the speedometer lever arm effect can be compensated for: the lever arm vector $\boldsymbol{L}$ was determined from these candidates by finding the best match between the GNSS and odometer-based speeds.

\section{Performance During GNSS Outages}

To investigate the error accumulation during GNSS outages, artificial gaps were injected into the GNSS position fix time series. The test trajectory, i.e., a round-trip drive in Aurora, and the start/end points of the four artificial data gaps are plotted in Figure 5. Two of the gaps were $38 \mathrm{~s}$ in duration whereas the other two spanned $85 \mathrm{~s}$. The gap durations and occurrence times were chosen arbitrarily. We examine two data sets: one where receiver was configured to use all four GNSS constellations but no correction data, and another one where the GNSS receiver was using GPS only with EGNOS corrections. Note that neither of these two GNSS processing configurations gives a $95 \%$ horizontal positioning accuracy better than $0.2 \mathrm{~m}$; however, the purpose of this test is to see the effect of the accuracy of the GNSS data on the error accumulation of sensor-based navigation.

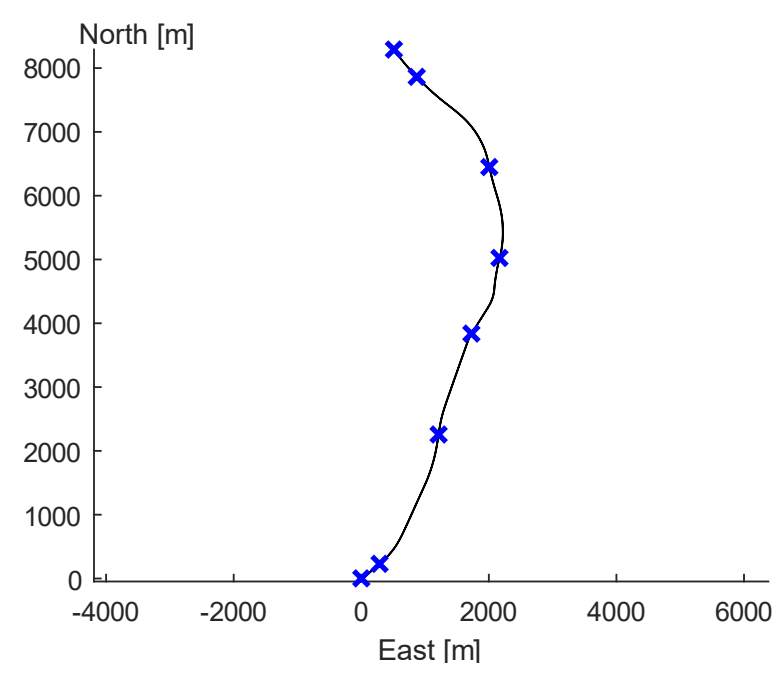

Figure 5 Test trajectory and the start/end points of the simulated GNSS outages (blue markers)

The position error accumulation in the multi-GNSS test is plotted in Figure 6. No GNSS correction data were used in this configuration, and the average position error is a couple of meters in total; clear spikes are observed during GNSS unavailability. In order to assess the effect of filter tuning on the results, we compare two solution variants: one obtained with nominal GNSS update weights, and another one where the GNSS measurements are given a lower weight (error standard deviation increased by a factor of 10) to increase the reliance on the error state evolution model. The different weightings produce somewhat different results, but the difference is not very remarkable. Thus, we conclude that the results are not severely affected by the choice of filter tuning within this scale. 

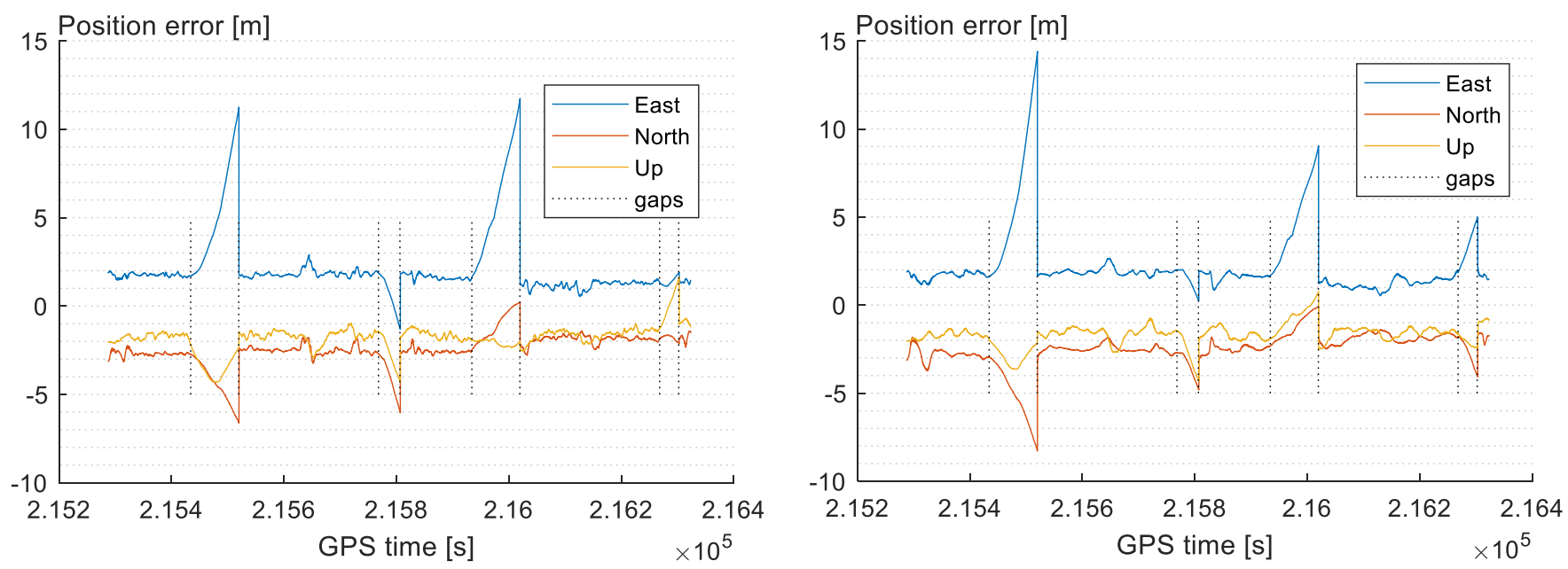

Figure 6 Position errors in the multi-GNSS test. Left: default weight on GNSS updates; right: reduced weight on GNSS updates

The accumulation of positioning errors in the case where the GNSS receiver is set to use EGNOS corrections is plotted in Figure 7. It can be seen that the accuracy of the EGNOS-based GNSS solution is better than in the multi-GNSS test, i.e., approximately $1 \mathrm{~m}$, but visible error accumulation is observed during the longer outages. Figure 7 also shows close-ups of the GNSS outage periods. The accumulated error spikes are lower than those in the multi-GNSS test, suggesting that the accuracy of GNSS updates is significant for the performance of the hybrid navigation algorithm. 

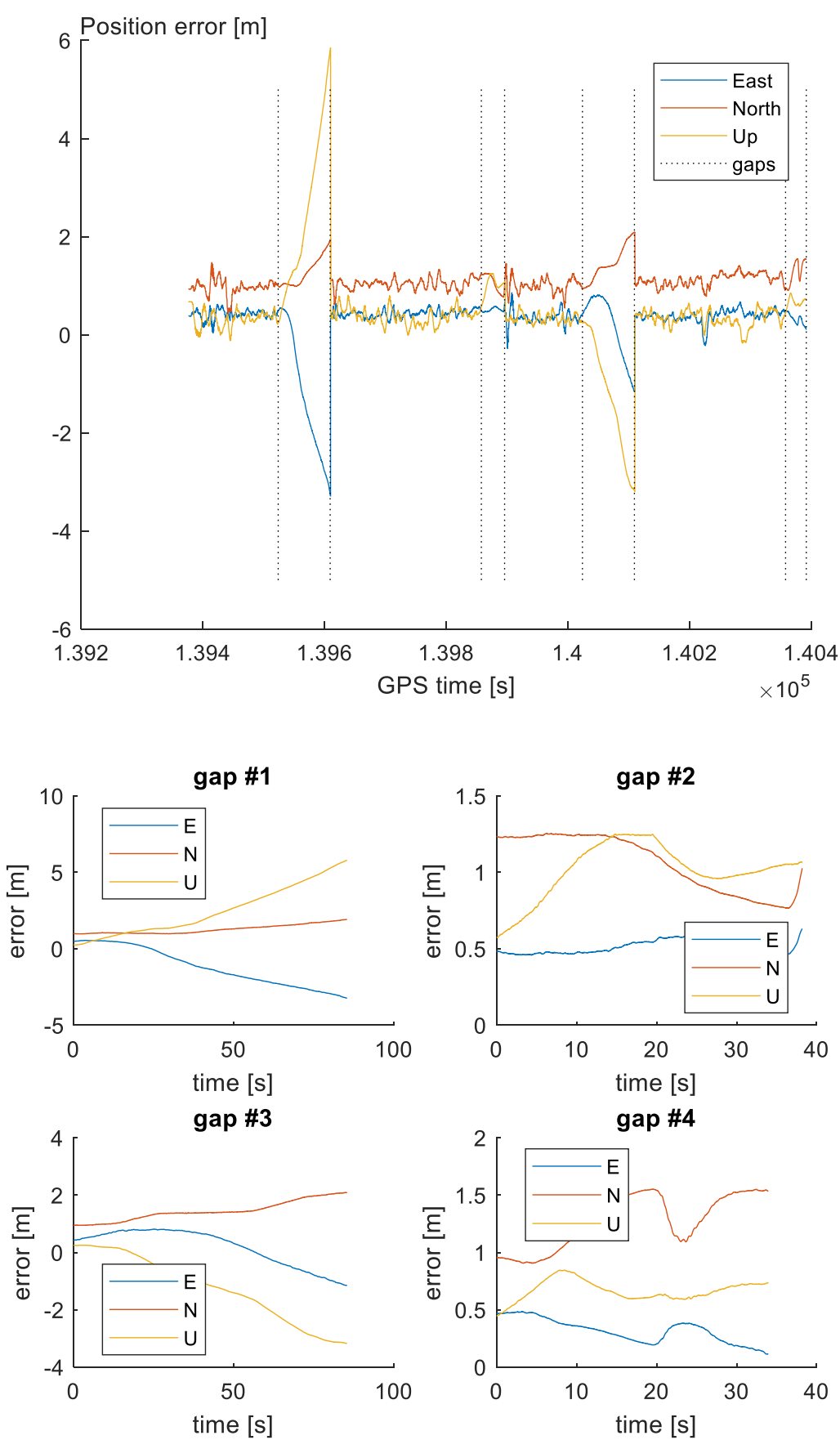

Figure 7 Position errors in the EGNOS test with artificial data gaps. Top: Entire time series; bottom: zoom to each GNSS outage period.

An important driver of position errors during GNSS outages are attitude estimation errors. The IMU of the reference system and the low-cost Xsens IMU were rigidly mounted but not mutually aligned. Consequently, one should expect to see constant offsets between the Euler angles estimated by the reference system and the proposed navigation algorithm. The Euler angle estimates with the two 
different weightings are compared in Figure 8. We observe a long-term variation in the roll angle estimates during the first half of the test data, but the coordinate differences converge to the same values for the latter half of the time. The solution with less weight on GNSS updates exhibits more noise on the Euler angles, but the long-term variations look similar. However, the choice of GNSS weighting makes no significant difference in the accumulation of attitude errors during the data gaps; this can be expected because both solutions were computed using the same gyroscope data.
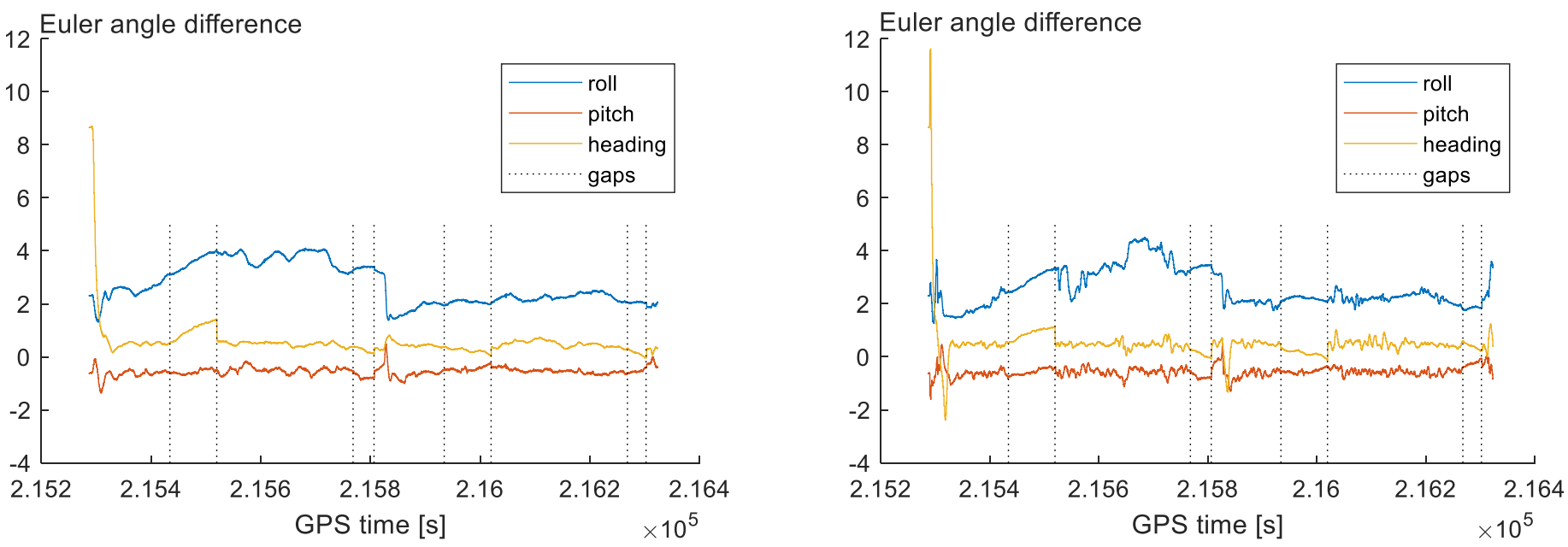

Figure 8 Difference of attitude estimates between the proposed algorithm and the reference system in the multi-GNSS test. Left: default weight on GNSS updates; right: reduced weight on GNSS updates.

In contrast, the attitude estimates obtained in the EGNOS test (Figure 9) follow the reference solution with no distinctive long-term variations, excluding the initial convergence period. The average values of the Euler angle offsets are similar to those obtained in the latter half of the multi-GNSS test, indicating that the attitude estimation works consistently.

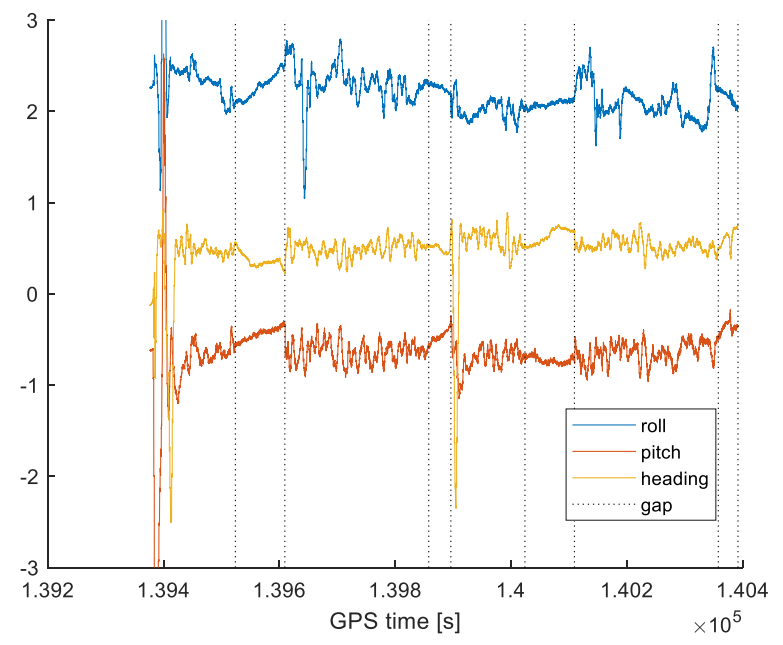

Figure 9 Difference of attitude estimates (in units of degrees) between the proposed algorithm and the reference system in the EGNOS test 


\section{Performance with Reduced GNSS Update Rate}

In this experiment, the GNSS updates were decreased from $1 \mathrm{~Hz}$ intervals to a slower rate in order to investigate the minimum GNSS sampling rate where a horizontal positioning accuracy better than $0.2 \mathrm{~m}$ could be maintained for $95 \%$ of the time. The GNSS position fixes utilized in this test were computed by means of RTK processing which can be expected to yield much more precise results than the stand-alone and EGNOS configurations employed in the two above experiments. As opposed to the experiments presented in the previous section, no prolonged GNSS outages were created but the sampling rate was decreased uniformly. The $0.2 \mathrm{~m}$ horizontal accuracy benchmark is chosen as a compromise between the requirements postulated in [2] and [3].
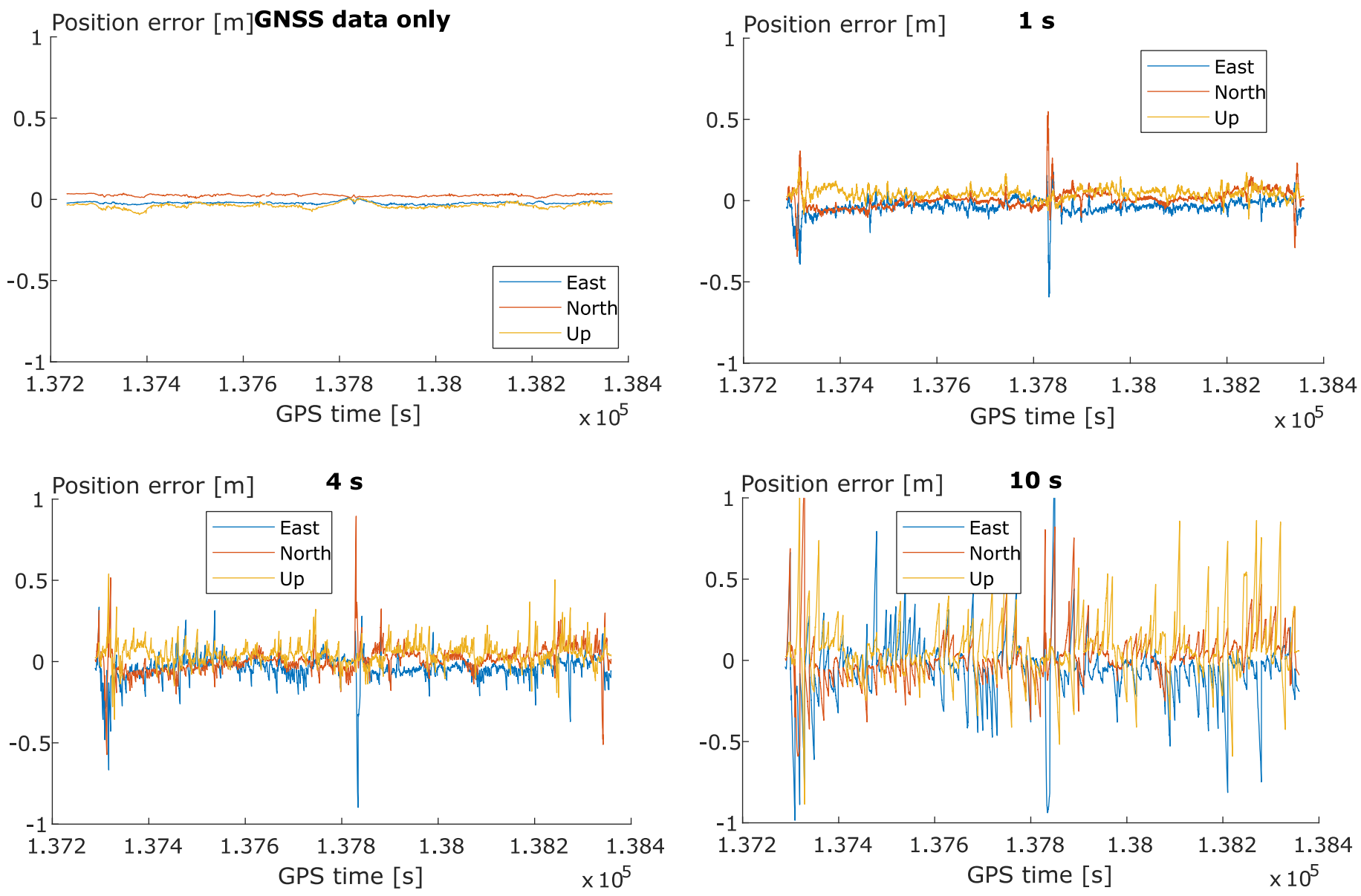

Figure 10 Position errors with different GNSS update rates. Top left: GNSS position only without sensors; top right: hybridization with $1 \mathrm{~s}$ update interval; bottom left: hybridization with $4 \mathrm{~s}$ update interval; bottom right: hybridization with $10 \mathrm{~s}$ update interval

The degradation of positioning accuracy as a function of GNSS update interval is depicted in Figure 10. It can be seen that the $1 \mathrm{~Hz}$ GNSS-only solution is very precise when compared with the high-rate hybrid solutions. The error accumulation between GNSS updates shows as spikes as the update rate gets sparser. 
Table 1 Horizontal positioning accuracy with different GNSS update intervals

\begin{tabular}{rrrr}
\hline Update interval [s] & \multicolumn{3}{c}{$95 \%$ position error [m] } \\
\cline { 2 - 4 } & Along-track & Cross-track & Horizontal \\
\hline 1 & 0.090 & 0.091 & 0.117 \\
2 & 0.102 & 0.116 & 0.140 \\
4 & 0.131 & 0.168 & 0.195 \\
5 & 0.146 & 0.202 & 0.230 \\
10 & 0.213 & 0.457 & 0.522 \\
\hline
\end{tabular}

The corresponding accuracy figures are shown in Table 1. It can be seen that four seconds was the maximum GNSS update interval where the $0.2 \mathrm{~m}$ horizontal accuracy goal could be met for $95 \%$ of the time. Furthermore, the table indicates that the cross-track error component increases faster than the along-track error, which is most probably caused by problems maintaining accurate attitude estimates with low-cost gyroscopes. Note that the $95 \%$ quantiles of along- and cross-track errors were computed separately, which is why their Euclidian norm is greater than the total horizontal $95 \%$ error quantile.

The maximum GNSS update interval of 4 seconds can be seen as short concerning the likelihood of GNSS outages due to environmental constructions (buildings or tunnels) or communications outages in the RTK correction data stream. Keeping in mind that different experiments cannot be directly compared because of differences in trajectory shape, speed, and sensor quality, the result seems to be at least in line with the study reported in [6] where meter-level errors were accumulated over $5 \mathrm{~s}$ GNSS update intervals when using a cell phone grade IMU and [7] where a position error smaller than $0.2 \%$ of the traveled distance was reported, implying a speed of $90 \mathrm{~km} / \mathrm{h}$ to accumulate $0.2 \mathrm{~m}$ of position error over 4 seconds.

\section{CONCLUSIONS}

This paper presented a hybrid navigation algorithm for validating the navigation infrastructure of the Aurora ecosystem. Based on low-cost inertial sensors and the on-board speedometer loosely coupled with a GNSS receiver, the algorithm can easily accommodate other sources of PNT for performance validation.

The performance of the algorithm during GNSS outages was analyzed. Using more accurate GNSS updates was observed to enable the system to keep the error accumulation slower after GNSS becomes unavailable. The test results indicated that with the current GNSS infrastructure available at the Aurora test bed, the maximum GNSS update period where the $95 \%$ horizontal positioning error could be maintained below $0.2 \mathrm{~m}$ was 4 seconds. While four seconds may not seem like a very long unavailability in the light of the well-known shortcomings of GNSS, the outcome was considered to be in line with results reported in existing studies. Nevertheless, it is evident that either higher quality sensors or further infrastructure is necessary to guarantee the navigation performance necessary for autonomous vehicles.

As future work, the added value of high-definition map information on the navigation performance during GNSS outages is to be investigated. Incorporating the map constraints into the hybridization algorithm is expected to require a more flexible filtering framework as the basis, such as a particle filter. However, as maps are available in the Aurora ecosystem, they should definitely be included in the validation of the benefits of Aurora for future autonomous vehicle experiments.

\section{ACKNOWLEDGMENTS}

This research was conducted within the Arctic-PNT project which has been technically and financially supported by the European Space Agency. The test data were collected using the experimental autonomous vehicle Martti developed by the RobotCar Crew of the VTT Technical Centre of Finland Ltd. 


\section{REFERENCES}

[1] European GNSS Agency, Report on Road User Needs and Requirements, issue 1.0, Oct. 2018

[2] COST Action TU1302, SaPPART Handbook: Assessment of positioning performance in ITS applications. Ifsttar, 2017. techniques et méthodes, TMI 2. ISBN 978-2-85782-727-6

[3] Y. Feng, C. Wang, and C. Karl, "Determination of Required Positioning Integrity Parameters for Design of Vehicle Safety Applications," in Proc. 2018 International Technical Meeting of the Institute of Navigation, Reston, VA, Jan. 2018, pp. 129141.

[4] G. Dissanayake, S. Sukkarieh, E. Nebot and H. Durrant-Whyte, "The Aiding of a Low-Cost Strapdown Inertial Measurement Unit Using Vehicle Model Constraints for Land Vehicle Applications," IEEE Transactions on Robotics and Automation, vol. 17, no. 5, Oct. 2001, pp. 731-747.

[5] H. Niedermeier, H. Beckmann, and B. Eissfeller, "Robust, secure and precise vehicle navigation system for harsh GNSS signal conditions," in Proc. 25th International Technical Meeting of The Satellite Division of the Institute of Navigation, Nashville, TN, Sep. 2012, pp. 1589-1600.

[6] A. Broumandan and G. Lachapelle, "Spoofing Detection Using GNSS/INS/Odometer Coupling for Vehicular Navigation," Sensors, Apr. 2018, doi:10.3390/s18051305

[7] T. Cozzens, "TDK offers Coursa Drive positioning software for autonomous vehicles," GPS World, Jan. 2019. Online: https://www.gpsworld.com/tdk-offers-coursa-drive-positioning-software-for-autonomous-vehicles/ [accessed 26 Jun 2019]

[8] Finnish Transport Infrastructure Agency, "E8 - Aurora, the Arctic intelligent transport test ecosystem", Feb. 2019. Online: https://vayla.fi/web/en/e8-aurora [accessed 6 Feb 2019]

[9] "First 5G Autonomous Car Driving Test Bed to Open in South Korea," News18, June 2019. Online: https://www.news18.com/news/tech/first-5g-autonomous-car-driving-test-bed-to-open-in-south-korea-2195933.html [accessed 27 Jun 2019]

[10] Millbrook Proving Ground, "Controlled Urban Test Bed for Connected and Autonomous Vehicles," 2019. Online: https://www.millbrook.co.uk/cav/ [accessed 27 Jun 2019]

[11] S. Thombre, S. Marila, M. Kirkko-Jaakkola, S. Honkala, M. Koivisto, H. Koivula, M. Z. H. Bhuiyan, and M. Petovello, ” What are the challenges to localization in autonomous cars in the Arctic?" Inside GNSS, Mar.-Apr. 2019, pp. 40-47.

[12] European GNSS Agency, EGNOS Data Access Service (EDAS) Service Definition Document, issue 2.2, Jun. 2019, doi: $10.2878 / 729922$

[13] H. Koivula, A. Laaksonen, S. Lahtinen, J. Kuokkanen, and S. Marila, "Finnish Permanent GNSS Network, FinnRef," in Proc. FIG Working Week 2017: Surveying the world of tomorrow - From digitalisation to augmented reality, Helsinki, Finland, May-June 2017. Available: https://www.fig.net/resources/proceedings/fig_proceedings/fig2017/papers/ts07c/TS07C_koivula_laaksonen_et_al_8939.pdf 